\title{
3D-QSAR of SIRT1 Activators Targeting Against Diet-Induced Metabolic Syndrome
}

\author{
Hye Ri Park, Kwang-su Park, Jina Choo, ${ }^{* *}$ and Youhoon Chong \\ Department of Bioscience and Biotechnology, Konktk Lniversity, Seoul 143-701, Korea. 'E-mail: chongvakonkuk acks \\ ${ }^{\dagger}$ College of Nursing, Korea Lnwersit, Seoul 136-705. Korea. ${ }^{*}$ E-mail: jinachoo'akorea ackr \\ Received Jine 17, 2009, Accepted Julv 6, 2009
}

Key Wonds: SIRT I Activator. Calorie restriction. Metabolic disease. 3D-QSAR

The prevalence of metabolic syndrome associated with increased risk for cardiovascular disease type 2 diabetes. or cancer has been increasing over the past decade. ${ }^{1.2}$ While traditional drug discovery efforts have been tackling these diseases by aiming at individual targets. recent studies in humans have suggested the possibility that the collection of the metabolic degenerative processes can be approached as a whole by controlling diet. especially calorie restriction. Studies of calorie restriction in a broad range of animals including primates, mice. worms, and yeast have suggested that a reduction in calorie intake lengthens lifespan and protects against cancer and other age-related diseases. ${ }^{3-5}$ There has therefore been much interest in developing pharmacological agents that mimic the effects of calorie restriction. Resveratrol a natural product derived from grapes, is the first reported mimetic of calorie restriction. Ever since the mode of action of resveratrol was elucidated to activate SIRT1 [sirtuin (silent mating type information regulation 2 homolog) 1] which. in turn. deacetylates p53 and promotes cell survival in a $\mathrm{NAD}^{+}$-dependent manner. numerous efforts have been devoted to discover novel activators of SIRT1. Through high throughput screening of a large selection of small molecules, Milne et al. identified three imidazothiazole derivatives as potent SIRT1 activators. which are structurally unrelated to but 1,000 -fold more active than resveratrol. Recently, in order to improve the potency as well as the solubility of the imidazothiazole derivatives two different libraries of analogues, imidazo[ $[1,2-b]$ thiazole ${ }^{8}$ and oxazolo $[4.5-b]$ pyridine. ${ }^{9}$ were constnicted and extensively investigated. The aim of the present study is to derive a predictive method for designing novel potent SIRTI activators through construction of 3D QSAR models.

For this purpose. from the literature ${ }^{8.9}$ SIRTI activation data of 33 imidazothiazole and oxazolopyridine derivatives were obtained (Fig. 1). At first, the 33 compounds were divided into two groups: 27 compounds as a training set and the other 6 compounds as a test set. The CoMFA ${ }^{10,11}$ (comparative molecular force analy sis) and CoMSIA ${ }^{1 \hat{1} .13}$ (comparative molecular similarity indices analy sis) methods were employed for deriving 3D-QSAR models consisting for a training set of 27 imidazothiazole and oxazolopy ridine derivatives. keeping in vitro activity as a dependent variable. The 3D-QSAR models were then validated using a test set of 6 compounds. which were not included in the development of the models. Finally, the contour plots of the 3D-QSAR model were analyzed to

${ }^{a}$ These two anthors contributed equally to this work provide helpful information on how to improve the potency of imidazothiazole as well as oxazolopyridine derivatives by structural modifications.

Tripos standard CoMFA field was used for construction of a COMFA model, whereas a CoMSIA model was built by using combinations of steric. electrostatic. hydrogen bond donor, hydrogen bond acceptor, and hydrophobic fields as descriptors. The reported $\mathrm{EC}_{3}$, values (concentration of a compound required to increase the enzyme activity by $50 \%$ ) of the training set as well as test set molecules, were converted into $\operatorname{logE} \mathrm{C}_{1.5}$ to describe the potency. CoMFA and CoMSIA were set at standard values (with default grid spacing of $2.0 \mathrm{~A}$ ), with a $s p^{3}$ carbon atom with one positive charge used to probe steric and electrostatic fields. The standard cutoff value was set to $30 \mathrm{kcal} / \mathrm{mol}$. As usual. PLS (partial least squares) method was used to establish and validate 3DQSAR, and LOO (leave-one-out) cross-validation method was used to evaluate the initial model. The cross-validated coefficient $q^{2}$ was calculated, the optimum number of components was then given and the 3D model was finally derived corresponding to the optimum number. The column filtering box was kept unchecked during all operations, and the results obtained from the PLS analy sis are summarized in Table 1.

A 3D-QSAR model is considered statistically significant if its $q^{2}$-value is larger than $0.3^{14}$ although a $q^{2}$-value larger than 0.4 to 0.5 is naturally preferable. The CoMFA model $q^{2}=0.486$. $N=5$ ) is consequently statistically significant, but the most stable and statistically significant 3D-QSAR model was provided by CoMSIA method using steric as well as electrostatic field as descriptor variables (CoMSIA $\left.A_{\mathrm{se}}\right)\left(q^{2}=0.626 . \hat{N}=5\right.$. Table 1). The CoMSIA field provided better model than the corresponding CoMFA fields presumably because the CoMSIA field was less affected by changes in molecular alignments particularly in this case which have diverse compounds with high conformational flexibilities. ${ }^{15}$ Other CoMSIA fields (hydrogen bond donor. acceptor, and hydrophobicity) either alone or in combination with CoMSIA se $_{s e}$ provided QSAR models with low statistical significance. which demonstrates that CoMSIA are sufficient and necessary to describe the activation of SIRT-I by imidazothiazole and oxazolopy ridine derivatives. The PLS analysis obtained above was used for the final non-cross-validated nun, giving good correlation coeffi-

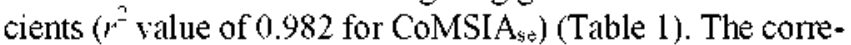
sponding field distributions of the steric and electrostatic field descriptor variables were 33.5 and $66.5 \%$, respectively. which indicates that electrostatic rather than steric field contributes 
<smiles>[R][Y]1ccc(C(=O)Nc2cc([R])cc(-c3nc4ncccc4o3)c2)cc1</smiles>

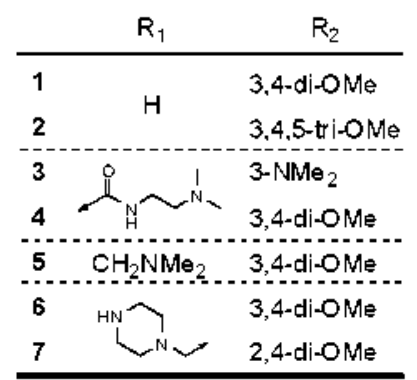<smiles>[R2]c1ccc(C(=O)Nc2cccc([R])c2)cc1</smiles>
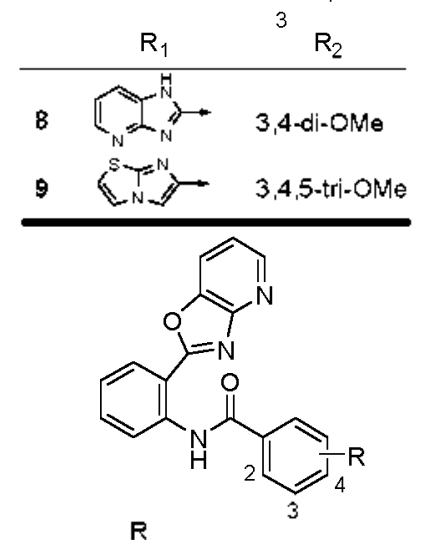

10 3,4-di-OMe

11 3-NMe2

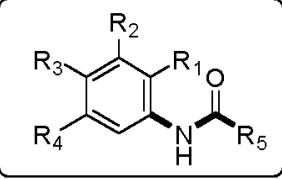<smiles>[R]C(=O)Nc1ccc([R])cc1-c1nc2ccccc2[nH]1</smiles>
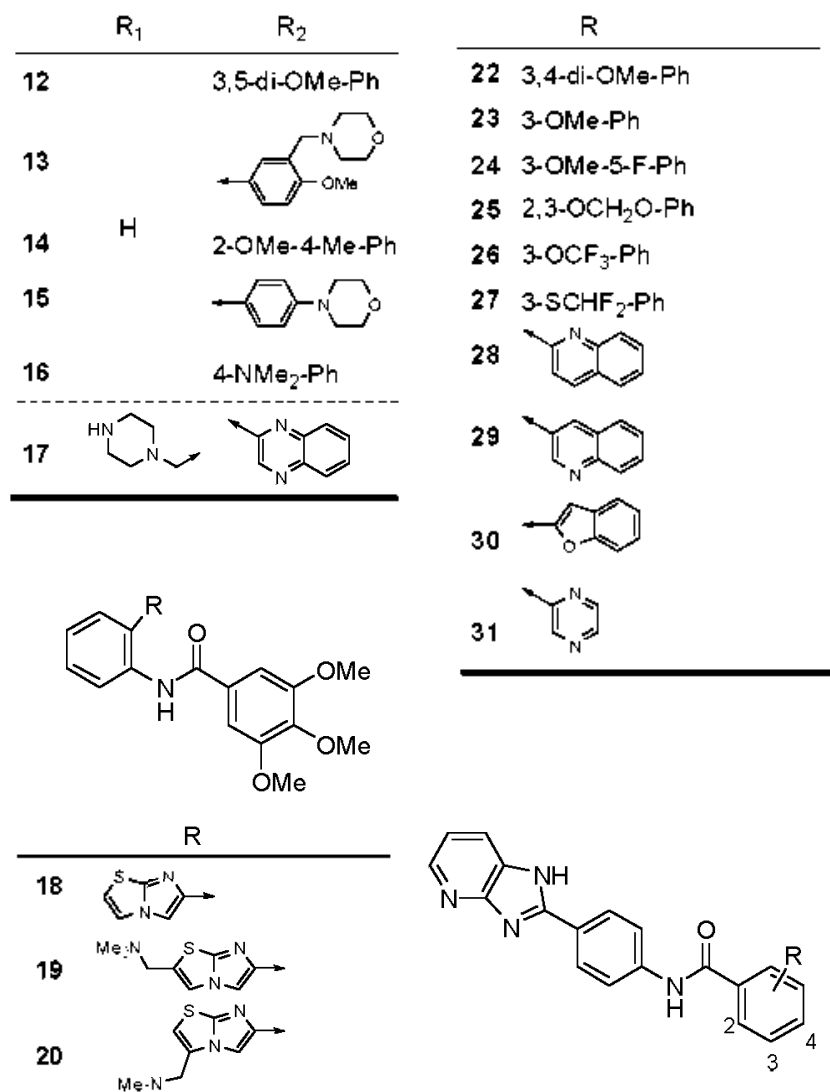

R

\section{2-OMe-4-Me}

33 3,4-di-OMe

Figure 1. Imidazothiazole and oxazolopyridine derivatives used for 3D-QSAR study. Fragments used for structural alignment are represented in bold lines.

Table 1. PLS analy sis on SIRTl activators

\begin{tabular}{|c|c|c|c|c|c|c|c|c|}
\hline \multirow{2}{*}{ Model } & \multicolumn{3}{|c|}{ Cross-Validated } & \multicolumn{3}{|c|}{ Non-cross-validated } & \multicolumn{2}{|c|}{ Fraction \% } \\
\hline & $\hat{q}$ & SEP & $N$ & $r^{2}$ & SEE & $\mathrm{F}$ & $S$ & $E$ \\
\hline CoMFA & 0.486 & 0.307 & 5 & 0.906 & 0.148 & 54.82 & 51.1 & 48.9 \\
\hline $\operatorname{CoMSLA}_{\mathrm{se}}{ }^{\sigma}$ & 0.626 & 0.257 & 5 & 0.982 & 0.058 & 182.09 & 33.5 & 66.5 \\
\hline $\operatorname{CoMSLA}_{\mathrm{da}}{ }^{b}$ & 0.037 & 0.391 & 2 & $-d$ & $-d$ & $-{ }^{d}$ & $-d$ & $-d$ \\
\hline $\operatorname{CoMSLA}_{13}{ }^{i}$ & 0.133 & 0.425 & 2 & $-{ }^{d}$ & $--^{d t}$ & $-{ }^{d}$ & $-d$ & $-{ }^{d}$ \\
\hline
\end{tabular}

$q^{2}$ : leave-one-out (LOO) cross-validated correlation coefficient. SEP: standard error of prediction. $N$ : aptimum number of components. $r^{2}:$ non-crossvalidated correlation coefficient. SEE: standard error of estimate, F: F-test value, S: fraction of steric field. E: fraction of electrostatic field. " $\mathrm{s}=$ steric field, e = electrostatic field. " $\mathrm{d}=$ hydrogen bond donor field, $a=$ hydrogen bond acceptor field. ${ }^{c} \mathrm{~h}=$ hydrophobic field. "not determined. 
Table 2. Companson of expermental (Exp) and CoMSIA modelpredicted (Pred) activation of Sirt- $1\left(\log \mathrm{EC}_{1}\right.$ s) by imidazothiazole and oxazolopyridine derivatives. Differences between the two values are represented by residuals $(\hat{0})$

\begin{tabular}{|c|c|c|c|c|c|c|c|}
\hline \multirow{2}{*}{ Compd- } & \multicolumn{2}{|c|}{$\log E C_{1.5}$} & \multirow{2}{*}{$\delta$} & \multirow{2}{*}{ Compd- } & \multicolumn{2}{|c|}{$\log E \mathrm{C}_{1.5}$} & \multirow{2}{*}{$\delta$} \\
\hline & Exp & Pred & & & Exp & Pred & \\
\hline 1 & -0.78 & -0.81 & 0.03 & 18 & -0.40 & -0.44 & 0.04 \\
\hline $2^{x}$ & -1.04 & -1.02 & -0.02 & 19 & -0.68 & -0.63 & -0.05 \\
\hline 3 & -0.26 & -0.29 & 0.03 & $20^{\circ}$ & -0.28 & -0.33 & 0.05 \\
\hline 4 & 0.15 & 0.14 & 0.01 & 21 & -0.51 & -0.55 & 0.04 \\
\hline 5 & -0.04 & 0.00 & -0.04 & 22 & -0.65 & -0.58 & -0.07 \\
\hline 6 & -0.04 & 0.00 & -0.04 & 23 & -0.57 & -0.43 & -0.14 \\
\hline 7 & 0.30 & 0.27 & 0.03 & 24 & -0.32 & -0.47 & 0.15 \\
\hline 8 & -0.15 & -0.14 & -0.01 & 25 & -0.69 & -0.71 & 0.02 \\
\hline 9 & -0.88 & -0.85 & -0.03 & $26^{*}$ & 0.05 & 0.08 & -0.03 \\
\hline 10 & 0.05 & 0.08 & -0.03 & $27^{*}$ & 0.29 & 0.31 & -0.02 \\
\hline 11 & -0.64 & -0.64 & 0.00 & 28 & 000 & 0.03 & -0.03 \\
\hline 12 & -0.23 & -0.23 & 0.00 & 29 & 0.17 & 0.14 & 0.03 \\
\hline 13 & 0.30 & 0.30 & 0.00 & 30 & 0.21 & 0.19 & 0.02 \\
\hline 14 & -0.61 & -0.61 & 0.00 & 31 & -0.23 & -0.24 & 0.01 \\
\hline $15^{\circ}$ & 0.05 & 0.06 & -0.01 & 32 & -0.36 & -0.40 & 0.04 \\
\hline 16 & 0.15 & 0.13 & 0.02 & $33^{\circ}$ & -0.20 & -0.21 & 0.01 \\
\hline 17 & 0.40 & 0.41 & -0.01 & & & & \\
\hline
\end{tabular}

test set compounds

more to the final CoMSIA model. Thus the electrostatic field plays crucial role in determining the activation of SIRT-1.

Predicted SIRT-1 activation. given as $\log \mathrm{EC}_{1}$ : values, and the residuals of the final non cross-validated COMSIA model are shown in Table 2, and the plot of actual versus predicted $\log \mathrm{EC}_{1.5}$ values is shown in Fig. 2.

The ultimate test for the usefulness of a 3D-QSAR model is predicting the activity of new compounds that are not included in the dataset used to obtain the model. To validate the stability and predictive ability of our 3D-QSAR model, 6 compounds (2. 15, 20, 26, 27, 33. Table 2) that were not included in the construction of CoMSIA model are selected as the test set. The binding affinities of the test set molecules were predicted reasonably well (residuals from -0.28 to 0.28 ) and the results are also summarized in Table 2 and Fig. 2.

Graphical representations of CoMSIA $A_{s e}$ steric and electro-
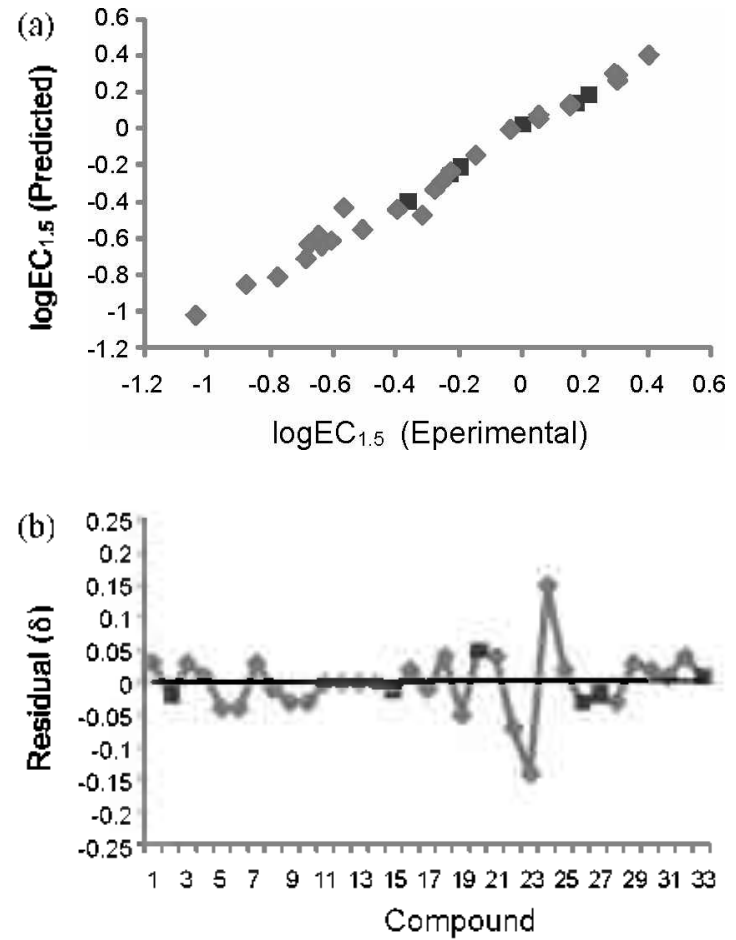

Figure 2. Plot of (a) experimental versus CoMSIA model-predicted $\log \mathrm{EC}_{1.5}$ of training set (diamond) and test set (rectangle); (b) Plot of residuals.

static field contour plots obtained by the field type "stDev*coeff" are displayed in Fig. 3. The contour maps were superinposed on 4 of which structure is representative of all other compounds used in this study. The green and blue regions in Fig. 3(a) and $3(\mathrm{~b})$ indicate areas where steric bulk and electronegative groups enhance biological activity. respectively. On the other hand. the yellow and red contours indicate regions where steric bulk and electronegative groups are detrimental to biological activity. Inuidazothiazoles and oxazopyridines. investigated in this study, have the central amide bond in common on both ends of which are linked to various aryl groups (enclosed by circle and rectangle in Fig. 3). The contour plot clearly shows that, among the two aryl groups, the one attached to the carbonyl carbon $(\mathrm{C}=\mathrm{O}$. enclosed by circle in Fig. 3 ) has linited scope of structural variation: the yellow steric contour confines the aryl group in a narrow region and the red and blue electrostatic (a)

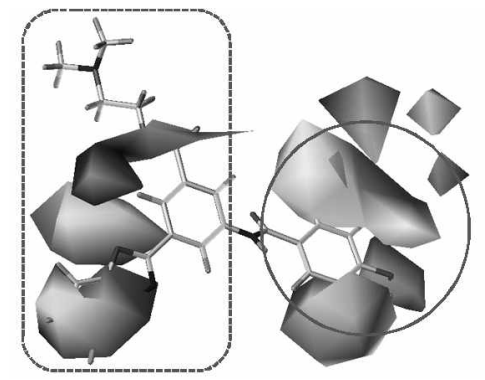

(b)

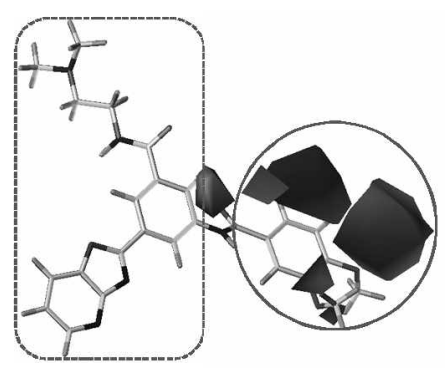

(c)

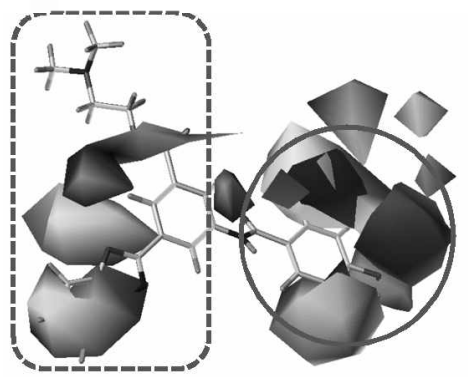

Figure 3. 3D contour maps around componnd 4 as the result of a CoMSLA analysis of the Sirt-1 activation by imidazothiazoles and oxazopyridines: (a) The color codings indicate regions where substitution enhances (green) or reduces (yellow) the Sirt-l activation: (b) Regions where electronegative substituents enhance (blue) or reduce (red) the Sirt-I activation; (c) Superimposed figure of (a) and (b). 
contours located side by side around the aryl ring allows only specific combinations of the aryl substituents. The aryl group on the other side of the amide linkage (attached to amide $-\mathrm{NH}$ and enclosed by rectangle in Fig. 3) is also sterically confined on one face of the aromatic ring but the other face near to the green steric contour is open for substitution with bulky substituents (Fig. 3a). It is of interest to note that no electrostatic contour can be found around this aryl group (Fig. 3b). Taken together, the contour plots illustrate that SIRTl activation of the imidazothiazole and oxazopyridine derivatives is under elaborate control by substituents on the aryl rings attached to both ends of the central amide linkage: The unfavorable steric as well as electrostatic contours around the aryl group attached to the amide carbonyl carbon significantly limit structural variation whereas various substituents can be introduced to the aryl group at the other side of the amide bond (amide $-\mathrm{NH}$ group). particularly around the green contours.

In summary, in order to derive a predictive method for designing and predicting the activities of novel SIRT-I activators. a 3D-QSAR model was constructed by using 27 known SIRT-1 activators. On the basis of common structural motifs ( $\mathrm{C}-\mathrm{NH}-$ $\mathrm{CO}-\mathrm{C}$ ) among the SIRT-1 activators a stable and predictive 3D-QSAR model with acceptable $q^{2}$-value was developed by using CoMSIA method with descriptors of steric and electrostatic field. The predictive ability of this model was successfilly validated by predicting the activity of 6 test set compounds exclusive to the training set. which indicates that the application of the COMSIA model for quantitative prediction of novel small molecule activators of SIRT-I would be feasible. The 3 D contour plot of the model also shows that. to improve SIRT-1 activation by imidazothiazole and oxazopyridine derivatives. extensive structural variation is anticipated on the aryl group directly attached to the central amide $-\mathrm{NH}$.

\section{Experimental Section}

All calculations were carried out on a linux enterprise operating syistem using molecular modeling software package SYBYL $v 7.2 .^{16}$ All compounds were constructed by the Sketch module in SYBYL base and assigned with Gästeiger-Hückel charges. For more flexible compounds. systematic searches were performed with an interval of $10^{\circ}$ on every rotatable bond to ensure their lowest energy conformations. Finally. they were minimized with Tripos force field. The most crucial step in performing 3D-QSAR is to determine the bioactive conformations of the compounds so that all compounds could be aligned together. In this study. the central amide moiety $[\mathrm{C}-\mathrm{NH}-\mathrm{CO}-$ $\mathrm{C}$. bold bonds in Fig. 1] commonly found in imidazothiazole and oxazolopyridine derivatives was used as the substructure for structural alignment. An oxazolopy ridine derivative (4) was used as a template for alignment from the alignment facility in SYBYL, and 27 training set molecules and 6 test set molecules were all aligned together.

Aclonowledgments. This work was supported by the Korea Science and Engineering Foundation (KOSEF) grant funded by the Korea government (MEST) (No. 2009-0071110). a grant from the Agenda Program (no. 200901OFT1 13068122), Rural Development Administration. Republic of Korea. and a grant from Biogreen 21 (Korea Ministry of Agriculture and Forestry). J. Choo was supported by a Korea University Grant (K0823601). KP is supported by the second Brain Korea 21.

\section{Refeiences}

1. Dekker, J. M; Giman, C; Giel, R; Nijpels, G.; Stehowwer, C. D. A.; Bouter, L. M.; Heine, R. T. Cinculation 2005, 112,666.

2. Russo, A.; Autelitano, M.: Bisanti, L. Em. J. Cancer 2008, 4t. 293.

3. Kenvon, C. Cell 2001, 105, 165.

4. Koubova, J:; Guarante, L. Genes Dev, 2003, 17, 313

5. Mattison, I. A.; Lane, M. A.; Roth, G. S.; Ingram, D. K. Exp. Gerontol. 2003, 38, 35 .

6. Howltz, K. T; Bitteman, K. T.; Cohen, H. Y.; Lamming, D. W; Lawu, S.; Wood, J. G.; Zipkin, R. E.; Chung, P ; Kisielewski, A.; Zhang, L.-L.: Scherer, B.; Sinclair, D. A. Nature 2003, 425,191 .

7. Milne, T. C.: Lambert, P. D.: Schenk, S.: Camey, D. P.; Smith, I. J.: Gagne, D. I.: Tin, L.; Boss, O.: Perni, R. B.: Vu, C. B.; Bemis, J. E.; Xie, R.; Disch, J. S.; Ng. P. Y.; Nunes, T. T.; Lynch, A. V; Yang, H.; Galonek, H.; Israelian, K. Chov, W.; Iffland, A.; Lavu, S.; Medvedik, O.; Sinclair, D. A.. Olefsky, J. M.: Jirousek, M. R.: Elliott. P. I.; Westphal, C. H. Natme 2007, $450,712$.

8. Vu, C. B.; Bemis, T. E.; Disch, J. S.; Ng, P. Y ; Nunes, J. J.; Milne, J. C. Camey, D. P.; Lynch, A. V.: Smith, J. T.; Lavu, S.; Lambert, P. D.: Gagne, D. J.: Jirousek, M. R:; Schenk, S. Olefsky, J. M.: Peni, R. B. J. Med. Chem. 2009, 52, 1275.

9. Bemis, T. E; Vu, C. B ; Xie, R; Numes, T. T. Ng. P. Y.; Disch, J. S.; Milne, T. C.; Carney, D. P.; Lynch, A. V.; Tin, L.; Smith, J. J.; Lavu, S.; Iftland, A, Jirousek, M. R, Peni, R. B. Bioorg. Med. Chem. Lett. 2009.19.2350.

10. Cramer, M.: Cramer, R. D; Jones, D. M. J. Am. Chem. Soc. $1988,110,5959$

11. Kim, J.; Lee, M.; Kang, S.-Y.; Park, J.; Lim, Y.; Koh, D.; Park, K. H.: Chong. Y. Bull Korean Chem. Soc. 2006, 27, 1025

12. Klebe, G.; Abraham, U.; Mietzner, T. J. hed. Chem. 1994, 37 , 4130 .

13. Kim, J.; Han, J. H.; Chong, Y. Bull. Korean Chem. Soc: 2006, 27 , 1919

14. Clark, M.: Cramer, R. D.: Jones, D. M.: Patterson, D. E:; Simeroth, P. E. Tenahedron Comput. Methodol 1990, $3,47$.

15. Klebe, G; Abraham, U.; Mietzner, T. J. Hed. Chem. 1994, 37 , 4130.

16. SYBYL 7.2 Tripos International, 1699 South Hanley Rd., St. Louls, Missouri, 63144, USA 